\title{
Broad Ligament Serous Adenocarcinoma
}

National Cancer Institute

\section{Source}

National Cancer Institute. Broad Ligament Serous Adenocarcinoma. NCI Thesaurus.

Code C126479.

A rare serous adenocarcinoma that arises from the broad ligament. 